\title{
Dynamics of transversally vibrating non-prismatic Timoshenko cantilever beams
}

\author{
N. Navadeh, R. W. Hewson, A. S. Fallah* \\ Department of Aeronautics, South Kensington Campus, Imperial College London, UK, SW7 2AZ
}

\begin{abstract}
The present study deals with evaluation of the dynamic response in a pulse loaded homogeneous non-prismatic Timoshenko cantilever beam. Subsequent to the derivation of the partial differential equations (PDE's) of motion using extended Hamilton's principle the eigenvalue problem has been set up and solved for eigenfrequencies and eigenfunctions. Galerkin's method of weighted residuals was applied to obtain governing ordinary differential equations (ODE's) for the system. The dynamic response under arbitrary pulse loading is obtained using the method of eigenfunction expansion which attributes to displacement and rotation fields generalised coordinates when the exact modes are chosen as shape functions. It has been shown that inclusion of few terms (in this case 5 ) in the series expansion provides a good correlation between the displacement fields and the truncated series. Dimensionless response parameters are introduced and two methods of non-dimensionalisation are proposed which could be useful in dealing with generic problems of a specified formulation.
\end{abstract}

Keywords:

Timoshenko beam, non-prismatic cantilever, Galerkin method, eigenfunction expansion method, non-dimensionalisation

\footnotetext{
* To whom correspondence should be addressed: Tel.: +442075945140, Email: as3@imperial.ac.uk (Arash S. Fallah)
} 


\section{Introduction}

As the level of functional requirements for structures elevates so does the level of sophistication in design. One such scenario emerges when the designer needs to optimise mass and stiffness distributions in a structural component. When 'skeletal' structural members are of concern or when idealisation as such is permissible this translates to using non-uniform beams. An airplane wing, a wind turbine blade, and a cantilever beam underneath a balcony are all examples of this class of structures. While sources of nonuniformity are numerous and could be ascribed to use of different materials or alteration of density along the length, the simplest case is that of geometric non-uniformity resulting in a homogeneous yet non-prismatic beam. Thus, the analysis of non-prismatic beams is of interest to aeronautical, mechanical, civil, biomedical and nuclear engineers.

Sometimes external pulse loads are exerted to this class of structures. When such components are subjected to time varying loads they respond dynamically, accordingly. Depending on the level of accuracy sought, different beam theories could be used to study transversal vibration of this class of structures. The problem of a vibrating beam can be formulated using the extended principle of least action (extended Hamilton's variational principle) and the governing partial differential equations and boundary conditions could be derived. The engineering theory considered in this work is the Timoshenko beam theory which is the most general beam theory as long as a plane deformation of a section is assumed. Other less general theories include Euler-Bernoulli, shear and Rayleigh beam theories. The differences among these theories are either due to kinematic assumptions made in reducing the three-dimensional continuum problem into a beam or in the inclusion of a term related to rotatory inertia [1]. There has been a comprehensive study conducted on the comparison of these formulations as applied to prismatic beams [2], nevertheless; no such study exists to date for non-prismatic beams.

While the engineering theories mentioned have been around since early $20^{\text {th }}$ century there has been a resurgence of interest in the topic of studying vibration in beams using these theories. There is a multitude of reasons for this but this is primarily due to requirements for certain applications which render a particular theory most suitable. Beam theories are used to study micro-vibration in micro-electromechanical systems (MEMS) [3], thermal excitations of high-frequency modes of cantilever vibrations [4], noise reduction using metamaterial beams and in reduction of magnetic noise in magnetic resonance force microscopy [5]. To mention but a few recent works, the vibration of a non-prismatic beam on an inertial elastic half-plane was studied using the Chebyshev's series approximation method [6], Timoshenko beam model was used in nanotechnology to study buckling and vibration of nanowires with surface effects [7] as well as multi-walled carbon nanotubes [8]. Rotating uniform Timoshenko beams were also studied using the theory $[9,10]$. Furthermore, recent studies have been conducted on rotating tapered Timoshenko beams [10-17] using the finite element method. Researchers have also introduced new basic displacement functions and have used them to develop finite elements for non-prismatic Timoshenko beams [18-20]. These studies are based on the development of beam elements and domain discretisation and are mostly specific to particular problems. 
In order to gain an understanding of vibration in systems idealised as Timoshenko beams the first step is to extract eigenvalues and eigenfunctions of the system. Eigenfunctions of the system form a complete set of functions through linear combination of which one can express any function satisfying the same essential boundary conditions. This fact is known as the linear expansion theorem.

There are differences between the models when it comes to estimation of natural frequencies and natural modes. Euler-Bernoulli model is known to slightly overestimate natural frequencies as it does not allow for shear relaxation and as a result renders the structure overstiff. This problem is exacerbated for the natural frequencies of higher modes and for nonslender beams. The predictions based on Rayleigh model only marginally improve those obtained based on the Euler-Bernoulli theory. Shear model adds shear distortion to EulerBernoulli model and improves accuracy in determination of natural frequencies considerably implying its effect is more pronounced than the effect of rotatory inertia [1]. Timoshenko's model is a major improvement as it includes both aforementioned effects. An important parameter in Timoshenko model is shape factor which is also known as the shear coefficient or area reduction factor. This parameter arises due to non-uniform distribution of shear over the cross section.

While many studies have been conducted on the derivation of equations of motion and finding eigenvalues and eigenfunctions as well as particular solutions using different beam theories no comprehensive study of general solution methods has been done on the subject involving non-prismatic Timoshenko beams. In the past, the dynamic response of nonprismatic beams has been investigated using numerical methods [21], variational principles [22], finite element method [18-20, 23], boundary element method [24], the transfer matrix method [25], Laplace transform method [26], and the dynamic stiffness method [27]. These studies ubiquitously require numerical implementation which could lead to large systems of equations and require user sophistication. Conversely, analytical derivation of eigenmodes and establishment of orthonormality condition rendered it possible to obtain transient response due to external load using the method of eigenfunctions expansion.

The present study describes the derivation and solution of equations of motion for a nonprismatic Timoshenko model as it is the most comprehensive of the engineering models mentioned above. In section 2, first the equations of motion and boundary conditions are derived for the Timoshenko beam model using extended Hamilton's principle. The eigenvalue problem i.e. the equations in the absence of the excitation term are then established. These equations are subsequently solved in section 3 for an example case using an analyticalnumerical approach proposed by researchers [28]. The eigenvalue problem makes it possible to obtain natural modes to be used at a later stage as exact shape functions in the eigenfunctions expansion method. The results are compared with a two-dimensional model in ABAQUS 6.14 and excellent agreement is observed. The method of weighted residuals (Galerkin) is then used to obtain the forced response for a non-prismatic beam under the action of a uniformly distributed pulse load of rectangular temporal pulse shape. Even though special spatial and temporal distributions are taken into account the formulation is generic and could be used to obtain similar results for other loading distributions. This is followed by 
a brief section on non-dimensional parameters and alternative approaches to solve the same problem and by conclusions in section 4 .

\section{Analyses}

\subsection{Derivation of the equations of motion}

This section deals with the derivation of equations of motion for a non-prismatic cantilever Timoshenko beam using extended Hamilton's principle. In this model both shear distortion and rotatory inertia effects are considered. Thus the strain energy is a function of both the angle of rotation and shear distortion and is obtained by adding the shear energy $\left(U_{S}\right)$ to bending energy $\left(U_{b}\right)$. Besides, kinetic energy, as an additive integral of the motion, is also obtained as the summation of translational $T_{t}$ and rotational $T_{r}$ terms. Therefore:

$$
\begin{aligned}
& U=U_{b}+U_{s}=\frac{1}{2} \int_{0}^{L} E I(x) \alpha^{\prime 2} d x+\frac{1}{2} \int_{0}^{L} k G A(x)\left(\frac{\partial w}{\partial x}-\alpha\right)^{2} d x . \\
& T=T_{t}+T_{r}=\frac{1}{2} \int_{0}^{L} \rho A(x)\left(\frac{\partial w}{\partial t}\right)^{2} d x+\frac{1}{2} \int_{0}^{L} \rho I(x) \dot{\alpha}^{2} d x .
\end{aligned}
$$

Where $w$ is the vertical displacement and $\alpha$ the rotation due to bending and $\rho, E, G, A, I$ and $k$ are density, Young modulus, shear modulus, cross sectional area, moment of inertia and shear coefficient, respectively. Through the application of the extended principle of least action (extended Hamilton's principle) one obtains the equations of motion as follows:

$$
\delta S=\int_{t_{1}}^{t_{2}} \delta(T-U) d t+\int_{t_{1}}^{t_{2}} \delta W_{n c} d t=0 .
$$

Where $S$ is the action integral and the work of non-conservative dynamic pulse load is obtained as follows:

$$
\delta W_{n c}=\int_{0}^{L} f(x, t) \delta w d x
$$

Hence:

$$
\begin{gathered}
\delta S=\int_{t_{1}}^{t_{2}} \int_{0}^{L}\left(\rho A(x) \dot{w} \delta \dot{w}+\rho I(x) \dot{\alpha} \delta \dot{\alpha}-E I(x) \alpha^{\prime} \delta \alpha^{\prime}-k G A(x)\left(w^{\prime}-\alpha\right)\left(\delta w^{\prime}-\delta \alpha\right)\right) d x d t \\
+\int_{t_{1}}^{t_{2}} \int_{0}^{L} f(x, t) \delta w d x d t=0 .
\end{gathered}
$$

Which upon integration by parts and imposition of the condition that at times $t_{1}$ and $t_{2}$ the mechanical state of the system is fully known it yields:

$$
\begin{aligned}
& \left(E I(x) \alpha^{\prime}\right)^{\prime}-\rho I(x) \ddot{\alpha}+k G A(x)\left(w^{\prime}-\alpha\right)=0, \\
& -\rho A(x) \ddot{w}+\left[k G A(x)\left(w^{\prime}-\alpha\right)\right]^{\prime}+f(x, t)=0 .
\end{aligned}
$$


And the corresponding boundary conditions are obtained as follows:

$$
\begin{aligned}
& {\left[\left(E I \alpha^{\prime}\right) \delta \alpha\right]_{0}^{L}=0,} \\
& {\left[k G A(x)\left(\alpha-w^{\prime}\right) \delta w\right]_{0}^{L}=0 .}
\end{aligned}
$$

Other beam theories can be obtained through exclusion of the terms from Eq.'s (1) and (2) ascribed to kinematical degrees of freedom non-existent in that model. This has been shown briefly in Appendix A. in Eq.'s (7a) and (7b) only one of the work conjugate parameters at a point on the boundary can be prescribed (either moment or rotation, either shear or displacement)

\subsection{Constructing the eigenvalue problem}

Solving for eigenvalues and eigenfunctions essentially provide the natural frequencies and modes of the non-prismatic Timoshenko beam. This is important due to two primary reasons: (1) It will render possible the evaluation of resonance frequencies, (2) It will let forced response be obtained as a linear combination of natural modes with time dependent coefficients.

By setting the excitation function to zero $(f(x, t)=0)$ the eigenvalue problem is constructed as follows:

$$
\begin{aligned}
& \left(E I(x) \alpha^{\prime}\right)^{\prime}-\rho I(x) \ddot{\alpha}+k G A(x)\left(w^{\prime}-\alpha\right)=0, \\
& -\rho A(x) \ddot{w}+\left[k G A(x)\left(w^{\prime}-\alpha\right)\right]^{\prime}=0,
\end{aligned}
$$

In order to formulate the problem in the most general form the method proposed by Lee and Lin [28] has been followed. Their study is based on dimensionless parameters of the model derived using Buckingham's Pi-theorem and use of non-dimensional groups to derive a single governing equation for the vibrating beam based on either transverse deformation or section rotation. The modes obtained as such will be used in a later section to obtain forced response vibration for the non-prismatic beam. Once the eigenvalue problem is established a harmonic admissible solution could be inserted into the equation to obtain the dynamic matrix.

Assuming the angle of rotation of the section and the flexural displacement are synchronised in time, as researchers have assumed [29] , and following the derivation of Lee and Lin [28] the two coupled partial differential equations of motion could be uncoupled. The derivation in the sequel reveals this condensation into the following uncoupled ordinary differential equations.

Assuming the solutions take the form:

$$
\begin{aligned}
& w(x, t)=Y(x) e^{i \omega t}, \\
& \alpha(x, t)=\psi(x) e^{i \omega t} .
\end{aligned}
$$

And defining the normalised spatial coordinate as $\xi=x / L$ all the spatial functions will be described as functions of $\xi$ rather than $x$. A set of non-dimensional parameters could be derived using Buckingham's Pi-theorem [30] as follows: 


$$
\begin{array}{cc}
q(\xi)=k G A(\xi) / k G A(0), & (10 a) \\
r(\xi)=E I(\xi) / E I(0), & (10 b) \\
s(\xi)=\rho A(\xi) / \rho A(0), & (10 c) \\
v(\xi)=J(\xi) / J(0), & (10 d) \\
\delta=E I(0) /\left(k G A(0) L^{2}\right), & (10 e) \\
\eta=J(0) /\left[\rho A(0) L^{2}\right], & (10 f) \\
\Omega^{2}=\rho A(0) \omega^{2} L^{4} / E I(0) . & (10 g)
\end{array}
$$

Using the non-dimensional set of parameters derived above the two governing differential equations can be re-cast in the following form [28]:

$$
\begin{gathered}
{\left[\left\{\frac{q(\xi)}{\delta}\right\}\left(y^{\prime}-\psi\right)\right]^{\prime}+s(\xi) \Omega^{2} y=0} \\
\left(r(\xi) \psi^{\prime}\right)^{\prime}+\left\{\frac{q(\xi)}{\delta}\right\}\left(y^{\prime}-\psi\right)+v(\xi) \eta \Omega^{2} \psi=0 . \\
\xi \in(0,1)
\end{gathered}
$$

Where $y=y(\xi)=Y(\xi) / L$ and $\psi=\psi(\xi)$ are functions of the dimensionless spatial variable $\xi$.

Eq. (11a) could be re-written as follows:

$$
\psi^{\prime}=\left(\frac{1}{q}\right)\left\{\left(q y^{\prime}\right)^{\prime}+\delta s \Omega^{2} y-q^{\prime} \psi\right\}
$$

Upon substitution of Eq. (12a) into the second fundamental Eq. (11b) one obtains:

$$
\psi=\frac{1}{\zeta}\left\{\frac{\delta r q^{\prime}}{q^{2}}-\left(\left(q y^{\prime}\right)^{\prime}+\delta s \Omega^{2} y\right)-\delta\left[\frac{r}{q}\left(\left(q y^{\prime}\right)^{\prime}+\delta s \Omega^{2} y\right)\right]^{\prime}-q y^{\prime}\right\}
$$

Where the function $\zeta$ is defined as follows:

$$
\zeta=\delta\left[r\left(\frac{q^{\prime}}{q}\right)^{2}-\left(\frac{r q^{\prime}}{q}\right)^{2}\right]+\delta \eta v \Omega^{2}-q .
$$

Thus the decoupled governing differential equations in terms of $y$ and $\psi$ are as follows: In terms of $y$ :

$$
\begin{gathered}
\left(\frac{q}{\zeta}\left\{\left(\frac{r q^{\prime}}{q^{2}}\right)\left[\left(q y^{\prime}\right)^{\prime}+\delta s \Omega^{2} y\right]-\left(\frac{r}{q}\right)\left[\left(q y^{\prime}\right)^{\prime}+\delta s \Omega^{2} y\right]^{\prime}-\left[r\left(\frac{q^{\prime}}{q^{2}}\right)-\left(\frac{r q^{\prime}}{q}\right)^{\prime}+v \eta \Omega^{2} y\right] y^{\prime}\right\}\right)^{\prime} \\
-s \Omega^{2} y=0
\end{gathered}
$$

And in terms of $\psi$ : 


$$
q\left\{\left[\frac{1}{s \Omega^{2}}\right]\left[\left(r \psi^{\prime}\right)^{\prime \prime}+\left(v \eta \Omega^{2} \psi\right)^{\prime}\right]\right\}^{\prime}+\delta\left(r \psi^{\prime}\right)^{\prime}+\left(\delta v \eta \Omega^{2}-q\right) \psi=0 .
$$

The boundary conditions are expressed as follows:

$$
\begin{gathered}
y(0)=0, \quad(14 a) \\
\psi(0)=0, \quad(14 b) \\
\psi^{\prime}(1)=0, \quad(14 c) \\
y^{\prime}(1)-\psi(1)=0 . \quad(14 d)
\end{gathered}
$$

The essential boundary conditions involve displacement and rotation as in Eq.'s (14a) and (14b) while the natural boundary conditions of Eq.'s (14c) and (14d) are on moment and shear, respectively.

Let $V_{i}(\xi)$ be a set of 4 linearly independent fundamental solutions of one of the corresponding governing characteristic differential equations above. The homogeneous solution is then described as follows:

$$
V(\xi)=\sum_{i=1}^{4} C_{i} V_{i}(\xi) .
$$

Where $C_{i}$ 's are constants to be determined using the boundary conditions.

The decoupled equations of motion reduce to the following cases for the case of a prismatic beam:

$$
\begin{aligned}
& y^{\prime \prime \prime \prime}+\Omega^{2}(\eta+\delta) y^{\prime \prime}+\left(\delta \eta \Omega^{4}-\Omega^{2}\right) y=0, \\
& \psi^{\prime \prime \prime \prime}+\Omega^{2}(\eta+\delta) \psi^{\prime \prime}+\left(\delta \eta \Omega^{4}-\Omega^{2}\right) \psi=0 .
\end{aligned}
$$

Which renders the general solution (fundamental functions) of the two equations the same as they are ODE's of exactly the same form.

Lee and Lin [31] derived the exact associated normalised fundamental solutions for the uniform Timoshenko beam resting on a uniform Winkler elastic foundation and possessing arbitrary semi-rigid boundary conditions. Through appropriate adjustment of parameters (i.e. by setting all parameters defined by Eq.'s (10a)-(10d) equal to unity) one can obtain the exact fundamental solutions for the prismatic cantilever beam. These are 4 functions encompassing trigonometric and hyperbolic trigonometric functions as follows:

$$
\begin{aligned}
& V_{1}=\frac{1}{\epsilon^{2}+\tau^{2}}\left(\tau^{2} \cosh (\epsilon \xi)+\epsilon^{2} \cos (\tau \xi)\right), \\
& V_{2}=\frac{1}{\epsilon^{2}+\tau^{2}}\left(\frac{\tau^{2}}{\epsilon} \sinh (\epsilon \xi)+\frac{\epsilon^{2}}{\tau} \sin (\tau \xi)\right), \\
& V_{3}=\frac{1}{\epsilon^{2}+\tau^{2}}(\cosh (\epsilon \xi)-\cos (\tau \xi)),
\end{aligned}
$$




$$
V_{4}=\frac{1}{\epsilon^{2}+\tau^{2}}\left(\frac{1}{\epsilon} \sinh (\epsilon \xi)-\frac{1}{\tau} \sin (\tau \xi)\right) .
$$

Where $\epsilon$ and $\tau$ are obtained as the roots of the characteristic equation as follows:

$$
\epsilon=\sqrt{\frac{-A+\sqrt{A^{2}-4 B}}{2}},(18 a) \quad \tau=\sqrt{\frac{A+\sqrt{A^{2}-4 B}}{2}} .
$$

Where $A$ and $B$ are derived as related to other system parameters as follows:

$$
\begin{aligned}
& A=\Omega^{2}(\eta+\delta), \\
& B=\Omega^{2}\left(\delta \eta \Omega^{2}-1\right) .
\end{aligned}
$$

It can be observed that each $V_{i}$ is a function of $\xi$ and $\Omega^{2}$ and could hence be written as $V_{i}\left(\xi, \Omega^{2}\right)$. Eq.'s (19a) and (19b) imply the existence of a threshold (or critical) frequency $\Omega_{c}=$ $1 / \sqrt{\eta \delta}$ above which the parameter $\epsilon$ is imaginary and a different scheme of obtaining eigenvalues and eigenfunctions must be implemented [2]. It must, nevertheless; be noted that the nature of solution does not alter.

It can be shown, rather easily, that if the slenderness ratio approaches zero the critical frequency $\Omega_{c}$ also approaches zero meaning the thick beam theory applies, while if the slenderness ratio approaches infinity so does the critical frequency, so thin beam theory is applicable. This is shown in Appendix B. The physical importance of this is that for a thin beam only very high modes are affected by the change in the solution.

It should be notes that the same shape functions as of the prismatic beam can be used to obtain the dynamic response of a non-prismatic beam. This is obvious from the following simple argument. Linear expansion theorem allows for using the natural modes of the nonprismatic beam to derive its dynamic response when subjected to an arbitrary pulse load ${ }^{\dagger}$. As the deformation of a beam is essentially described by a linear combination of a complete set of independent functions satisfying the essential boundary conditions any natural mode of the non-prismatic beam is a linear combination of natural modes of the prismatic one. And as the natural modes of the prismatic beam are linear combinations of functions (17a)-(17d) the same base functions can be used to derive the eigenfunctions of the non-prismatic beam and therefore can be utilised through the method of eigenfunction expansion to yield the correct dynamic response under generalised pulse loading scenario for the non-prismatic beam. It must be, however, mentioned that the natural modes of the non-prismatic beam do not necessarily form an orthogonal set. This is proved in Appendix C.

If the four fundamental modes are normalised as follows then subsequent to substituting the solution into the associated boundary conditions the associated frequency equation is obtained.

\footnotetext{
${ }^{+}$Arbitrary in this context refers to both spatial and temporal distributions.
} 


$$
\left[\begin{array}{cccc}
V_{1}(0) & V_{2}(0) & V_{3}(0) & V_{4}(0) \\
V_{1}^{\prime}(0) & V_{2}^{\prime}(0) & V_{3}^{\prime}(0) & V_{4}^{\prime}(0) \\
V_{1}^{\prime \prime}(0) & V_{2}^{\prime \prime}(0) & V_{3}^{\prime \prime}(0) & V_{4}^{\prime \prime}(0) \\
V_{1}^{\prime \prime \prime}(0) & V_{2}^{\prime \prime \prime}(0) & V_{3}^{\prime \prime \prime}(0) & V_{4}^{\prime \prime \prime}(0)
\end{array}\right]=\left[\begin{array}{llll}
1 & 0 & 0 & 0 \\
0 & 1 & 0 & 0 \\
0 & 0 & 1 & 0 \\
0 & 0 & 0 & 1
\end{array}\right]
$$

\section{Solving for the response}

\subsection{Solving for eigenfrequencies and eigenmodes}

The roots of the determinant of the dynamic matrix, the entries of which are functions of $\Omega^{2}$, were solved using a code written in Matlab. The detailed derivation of terms are given in Lee and Lin [28] and will not be repeated here. The frequency equation (determinant of the dynamic matrix) for dimensionless flexural displacement is as follows:

$$
\begin{gathered}
\pi_{w}=F_{22} G_{33} G_{44}+F_{23} G_{34} G_{42}+F_{24} G_{32} G_{43}-F_{22} G_{32} G_{43}-F_{23} G_{32} G_{44}-F_{24} G_{33} G_{42} \\
=0 .(21)
\end{gathered}
$$

Where the terms of the determinant as derived as follows:

$$
\begin{gathered}
F_{22}=B_{1}(0), \quad(22 a) \\
F_{23}=B_{2}(0), \quad(22 b) \\
F_{24}=B_{3}(0), \quad(22 c) \\
F_{41}=q B_{1} /\left.\delta\right|_{\xi=1}, \quad(22 d) \\
F_{42}=q\left(B_{1}-\zeta\right) /\left.\delta\right|_{\xi=1}, \quad(22 e) \\
F_{43}=q B_{2} /\left.\delta\right|_{\xi=1}, \quad(22 f) \\
G_{3 j}=F_{34} V_{j}^{\prime \prime \prime}(1)+F_{33} V_{j}^{\prime \prime}(1)+F_{32} V_{j}^{\prime}(1)+F_{31} V_{j}(1), \\
G_{4 j}=F_{44} V_{j}^{\prime \prime \prime}(1)+F_{43} V_{j}^{\prime \prime}(1)+F_{42} V_{j}^{\prime}(1)+F_{41} V_{j}(1) .
\end{gathered}
$$

Where $V_{j}(\xi)$ are defined by Eq.'s (17a)-(17d), and $B_{j}(\xi)$ as follows:

$$
\begin{aligned}
& B_{0}(\xi)=\delta^{2} s \Omega^{2}\left(2 r q^{\prime}-q r^{\prime}\right) / q^{2}-s^{\prime} r \Omega^{2} / q, \\
& B_{1}(\xi)=\delta q^{\prime}\left(2 r q^{\prime}-q r^{\prime}\right) / q^{2}-\left[q^{\prime \prime}+\delta s r \Omega^{2} / q\right]-q, \\
& B_{2}(\xi)=\delta\left(2 r q^{\prime}-q r^{\prime}\right) / q-2 r q^{\prime} / q, \\
& B_{3}(\xi)=-\delta r .
\end{aligned}
$$

Similar formulation is possible in terms of angle of rotation $\pi_{\alpha}=0$ and the details are provided in Lee and Lin [28] (see Appendix II of that work). The frequencies obtained based on angle of rotation are exactly the same as those obtained by solving the eigenvalue problem for displacement function. This is anticipated as the two were assumed to be synchronised (See Eq.'s (9a) and (9b)).

A case study has been conducted for a non-prismatic beam of dimensions shown as in Fig. 1 . The eigenvalues obtained based on the displacement and rotation equations are 
corroborated with those obtained using Finite element software package ABAQUS 6.14. Table 1 shows a correlation of the results.

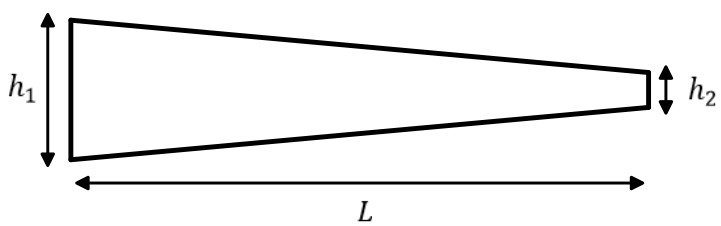

Figure 1: A non-prismatic two-dimensional cantilever beam of density $\rho=7800 \mathrm{~kg} / \mathrm{m}^{3}$, modulus of elasticity $E=2 E 11 \mathrm{~N} / \mathrm{m}^{2}$ and Poisson's ratio $v=0.3$. Dimensions of the beam are $h_{1}=10 \mathrm{~m}, h_{2}=$ $5 \mathrm{~m}, L=100 \mathrm{~m}$ simulated in ABAQUS using fine converged mesh of reduced integration quadratic plane stress (CPS8R) elements.

While the formulation is generic and allows for any change in the cross sectional dimensions and properties, the considered case is a simple one with linear change in the depth of the beam where $\rho$ and $E$ are constants and $A(x)$ and $I(x)$ are determined by the non-prismatic geometry and for the specific case considered are defined as follows:

$$
\begin{aligned}
& A(x)=b\left(h_{1}+\left(\frac{h_{2}-h_{1}}{L}\right) x\right) \\
& I(x)=\frac{b}{12}\left(h_{1}+\left(\frac{h_{2}-h_{1}}{L}\right) x\right)^{3}
\end{aligned}
$$

Where $b=1$, and $L, h_{1}$ and $h_{2}$ are constants the values of which are given in the caption of Fig. 1.

\begin{tabular}{|c|c|c|c|}
\hline Mode number & Frequency (analytical) & Frequency (FEM) & Percentage of error (\%) \\
\hline 1 & 0.87 & 0.885 & 2.16 \\
\hline 2 & 4.18 & 4.14 & 0.97 \\
\hline 3 & 11.21 & 10.28 & 9.05 \\
\hline 4 & 20.02 & 18.751 & 6.74 \\
\hline 5 & 31.08 & 29.05 & 7.00 \\
\hline 6 & 44.27 & 40.74 & 8.66 \\
\hline 7 & 58.17 & 53.46 & 8.82 \\
\hline 8 & 72.53 & 66.96 & 8.32 \\
\hline
\end{tabular}

Table 1: Comparison of modal natural frequencies obtained from the analytical model and finite element package ABAQUS 6.14

Natural modes derived based on the analytical model for the first 8 modes of vibration are also shown schematically in Fig. 2. Natural modes obtained from finite element software ABAQUS 6.14 are shown in Fig. 3. 


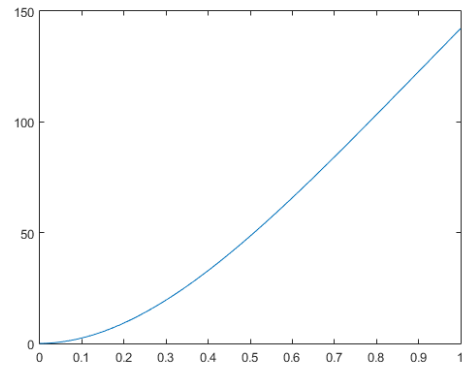

Mode 1

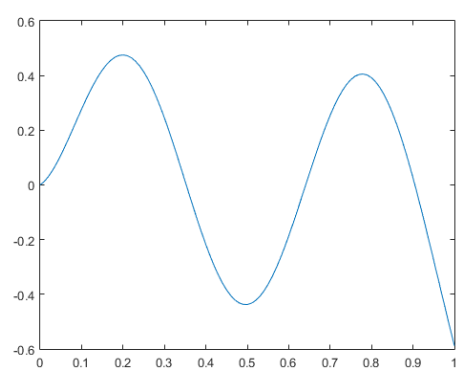

Mode 4

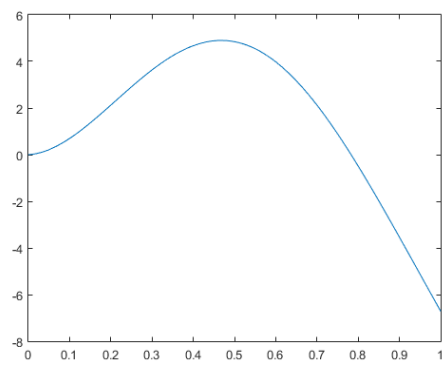

Mode 2

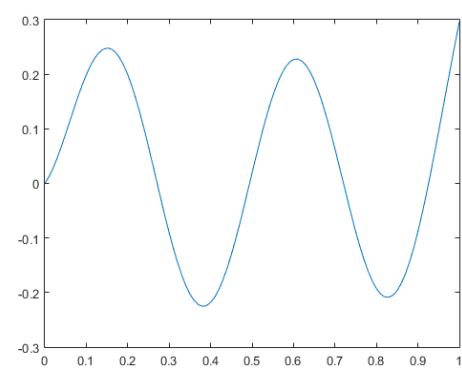

Mode 5

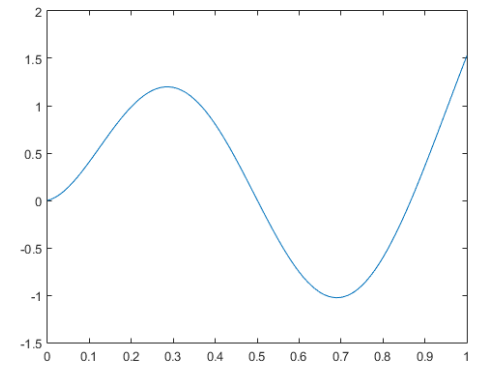

Mode 3

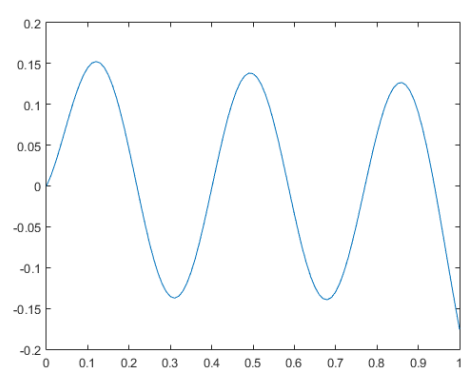

Mode 6

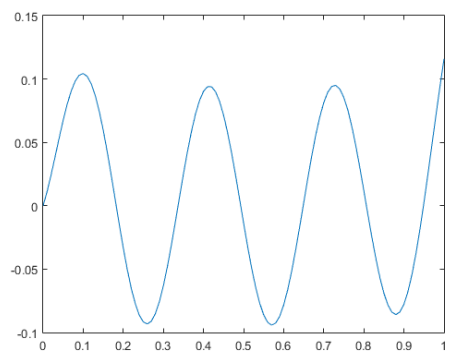

Mode 7

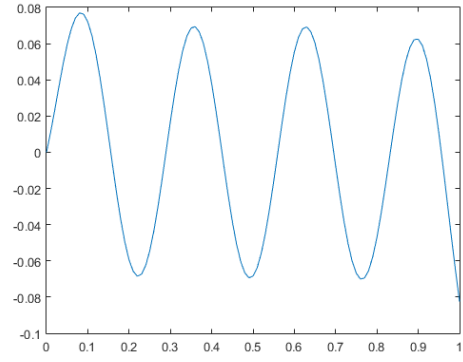

Mode 8

Figure 2: Schematics of natural modes extracted from the analytical model

As it could be seen the number and spacing of nodes and anti-nodes in the two models correspond well to each other. It must be noted that as a 2D model has been set up and run in ABAQUS there are natural modes associated with axial deformation of the beam. These are not captured in the current study as the analytical formulation for the Timoshenko beam does not allow for axial deformation of the beam neutral axis. Three such modes are shown in Fig. 4.

Once the eigenvalues and eigenmodes are derived it is a relatively simple task to obtain free and force vibration response for the system when subject to external excitation or as an initial value problem. The procedure to obtain free and forced vibration responses is as explained in the sequel. 
Mode 1

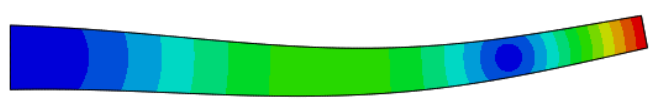

Mode 2

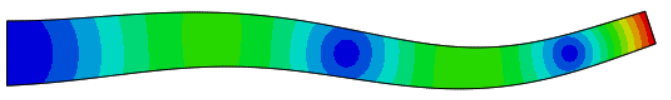

Mode 3

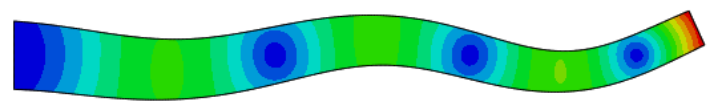

Mode 4

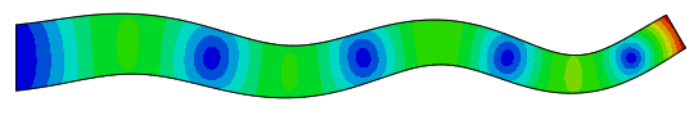

Mode 5

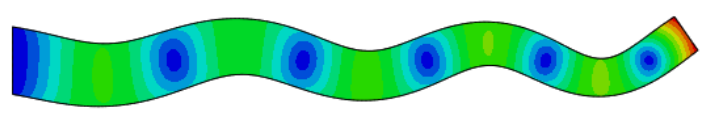

Mode 6

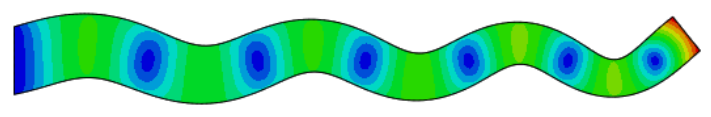

Mode 7

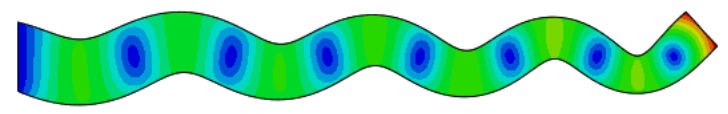

Mode 8

Figure 3: Natural modes extracted from the finite element software (ABAQUS)-the blue regions signify nodes and the green/red regions anti-nodes 
Axial mode 1

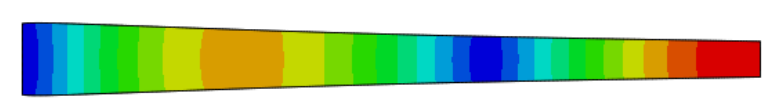

Axial mode 2

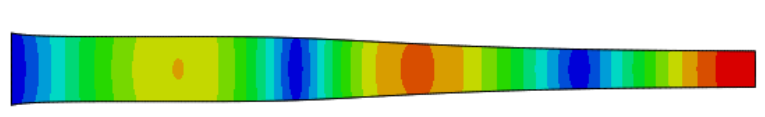

Axial mode 3

Figure 4: The first three axial modes of vibration (not considered in the present study as inextensible beam theory is used)- the blue regions signify nodes and the green/red regions anti-nodes

\subsection{Dynamic response}

As mentioned before, the method of eigenfunctions expansion allows expressing the dynamic response as a linear function of natural modes (eigenfunctions) as follows:

$$
\begin{gathered}
y(\xi, t)=\sum_{i=1}^{N} W_{i}(t) \Phi_{i}\left(\xi, \Omega^{2}\right), \\
\psi(\xi, t)=\sum_{i=1}^{N} \Theta_{i}(t) \Psi_{i}\left(\xi, \Omega^{2}\right) .
\end{gathered}
$$

Where for each mode the non-dimensional frequency is a fixed number and in what follows we shall depict the natural modes as $\Phi_{i}(\xi)$ and $\Psi_{i}(\xi)$, representing them for displacement and rotation, respectively; as a function of the spatial coordinate $\xi$ only. The functions $W_{i}(t)$ and $\Theta_{i}(t)$ represent generalised coordinates as temporal functions. Given both $\Phi_{i}(\xi)$ and $\Psi_{i}(\xi)$ are linear functions of $V_{j}\left(\xi, \Omega^{2}\right)$, as the orthogonal base functions which span the entire set of smooth functions defined over the domain, by substituting Eq.'s (25a) and (25b) into Eq.'s (6a) and (6b) and considering non-dimensional spatial part as Eq.'s (11a) and (11b) through pre-multiplication of a weighting function $\chi_{\alpha}$ (as in (26)) and integration on the entire domain and by using the weighted residuals method one can obtain the Eq.'s (27a) and (27b) as equations of motion i.e. ODE's in terms of generalised coordinates.

$$
\begin{gathered}
\int_{0}^{1} \chi_{\alpha}\left(\sum_{j=1}^{N} L_{i j} u_{j}+Q_{i}\right) d \xi=0 . \quad(\alpha, i=1,2) \\
L_{11}^{*} y+L_{12}^{*} \psi+Q_{1}^{*}=0 \\
L_{21}^{*} y+L_{22}^{*} \psi+Q_{2}^{*}=0 .
\end{gathered}
$$


Where, in (26), $L_{i j}$ are differential operators comprising spatial as well as temporal derivative operators, $\chi_{\alpha}$ the weighting functions and $u_{j}$ represent functions $y$ and $\psi$ i.e. $u_{1}=y$ and $u_{2}=\psi$. The operators and generalised forces in Eq.'s (27a) and (27b) are re-defined in a similar fashion, for instance $Q_{i}^{*}=\int_{0}^{1} \chi_{\alpha} Q_{i} d \xi$.

By taking $\chi_{\alpha}=\Phi_{\alpha}(\xi)$ or $\chi_{\alpha}=\Psi_{\alpha}(\xi)$ we arrive at Galerkin's method. Thus:

$$
\begin{aligned}
& \sum_{i=1}^{N}\left(M_{i j}^{(1)} \ddot{\Theta}_{i}+K_{i j}^{(1)} \Theta_{i}+R_{i j}^{(1)} W_{i}\right)=0, \\
& \sum_{i=1}^{N}\left(M_{i j}^{(2)} \ddot{W}_{i}+K_{i j}^{(2)} W_{i}+R_{i j}^{(2)} \Theta_{i}+F_{j}^{(2)}\right)=0 .
\end{aligned}
$$

Where the coefficients corresponding to generalised mass $M_{i j}^{(r)}$, generalised stiffness $K_{i j}^{(r)}$ as well as the generalised force $F_{j}^{(2)}$ are determined as follows:

$$
\begin{gathered}
M_{i j}^{(1)}=-\int_{0}^{1} \rho I \Psi_{i} \Psi_{j} d \xi \\
K_{i j}^{(1)}=\int_{0}^{1} \Psi_{j}\left(E I \Psi_{i}^{\prime \prime}+(E I)^{\prime} \Psi_{i}^{\prime}-k G A \Psi_{i}\right) d \xi, \\
R_{i j}^{(1)}=\int_{0}^{1} k G A \Phi_{i}^{\prime} \Psi_{j} d \xi, \\
M_{i j}^{(2)}=-\int_{0}^{1} \rho A \Phi_{i} \Phi_{j} d \xi, \\
K_{i j}^{(2)}=\int_{0}^{1}\left((k G A)^{\prime} \Phi_{i}^{\prime} \Phi_{j}+k G A \Phi_{i}^{\prime \prime} \Phi_{j}\right) d \xi, \\
R_{i j}^{(2)}=-\int_{0}^{1}\left((k G A)^{\prime} \Psi_{i} \Phi_{j}+k G A \Psi_{i}^{\prime} \Phi_{j}\right) d \xi, \\
F_{j}^{(2)}=\int_{0}^{1} \Phi_{j} f(\xi, t) d \xi .
\end{gathered}
$$

Where the spatial derivatives of system parameters are derived using the finite difference method. With the model parameters and the force function defined the only remaining step would be to determine the initial conditions in terms of generalised coordinates. This is done using the procedure explained in the sequel. Using (25) one obtains the relation between displacement and rotation fields and the generalised coordinates and generalised velocities as follows:

$$
y(\xi, 0)=\sum_{i=1}^{N} W_{i}(0) \Phi_{i}(\xi)
$$




$$
\begin{array}{r}
\psi(\xi, 0)=\sum_{i=1}^{N} \Theta_{i}(0) \Psi_{i}(\xi), \\
\dot{y}(\xi, 0)=\sum_{i=1}^{N} \dot{W}_{i}(0) \Phi_{i}(\xi), \\
\dot{\psi}(\xi, 0)=\sum_{i=1}^{N} \dot{\Theta}_{i}(0) \Psi_{i}(\xi) .
\end{array}
$$

Then each equation could be pre-multiplied by the relevant mode and integrated over the entire domain leading to generalised initial conditions. For instance, for Eq. (30a) the procedure is as follows:

$$
\Phi_{j}(\xi) y(\xi, 0)=\sum_{i=1}^{N} W_{i}(0) \Phi_{j}(\xi) \Phi_{i}(\xi),
$$

Thus:

$$
\int_{0}^{1} \Phi_{j}(\xi) y(\xi, 0) d \xi=\int_{0}^{1} \sum_{i=1}^{N} W_{i}(0) \Phi_{j}(\xi) \Phi_{i}(\xi) d \xi,
$$

Which yields:

$$
\sum_{i=1}^{N} \Gamma_{i j} W_{i}(0)=\Xi_{j}
$$

Where $\Gamma_{i j}=\int_{0}^{1} \Phi_{j}(\xi) \Phi_{i}(\xi) d \xi$ and $\Xi_{j}=\int_{0}^{1} \Phi_{j}(\xi) y(\xi, 0) d \xi$ so:

$$
\{W(0)\}=[\Gamma]^{-1}\{\Xi\}
$$

Similarly for generalised velocity:

$$
\{\dot{W}(0)\}=[\Gamma]^{-1}\{\Pi\}
$$

Where $\Pi_{i}=\int_{0}^{1} \Phi_{i}(\xi) \dot{y}(\xi, 0) d \xi$. For rotations one must use the shape functions $\Psi_{i}(\xi)$ and similar results could be obtained.

It must be mentioned that since eigenmodes are not orthogonal in the general case (See Appendix C) the coefficient matrices constructed using the procedure explained above are not in general diagonal thus the equations cannot be uncoupled. If the modes, however, were orthogonal the following simple relations would provide the initial conditions for the ODE's in terms of generalised coordinates and no matrix inversion were required. 


$$
\begin{gathered}
W_{i}(0)=\frac{\int_{0}^{1} \Phi_{i}(\xi) w(\xi, 0) d \xi}{\int_{0}^{1} \Phi_{i}^{2} d \xi}, \\
\Theta_{i}(0)=\frac{\int_{0}^{1} \Psi_{i}(\xi) \alpha(\xi, 0) d \xi}{\int_{0}^{1} \Psi_{i}^{2} d \xi} \\
\dot{W}_{i}(0)=\frac{\int_{0}^{1} \Phi_{i}(\xi) \dot{w}(\xi, 0) d \xi}{\int_{0}^{1} \Phi_{i}^{2} d \xi} \\
\dot{\Theta}_{i}(0)=\frac{\int_{0}^{1} \Psi_{i}(\xi) \dot{\alpha}(\xi, 0) d \xi}{\int_{0}^{1} \Psi_{i}^{2} d \xi} .
\end{gathered}
$$

As the integration is over space while differentiation is with respect to time there is essentially no difference in the procedure between the determination of initial generalised coordinate and initial generalised velocity for each generalised coordinate.

For the sake of analyses in this section a rectangular pulse shape with uniform distribution has been considered. The temporal and spatial distributions of the pulse shape are depicted in Fig. 5.

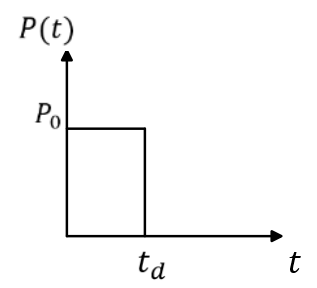

(a)

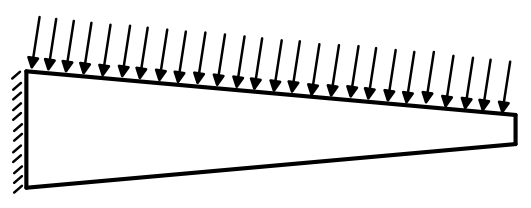

(b)

Figure 5: (a) temporal distribution, (b) spatial distribution, of the pulse load

It is obvious that in the analytical model the uniformly distributed load (UDL) must have the intensity of $P_{0}$. This is since the total vertical load on the upper surface is $P_{0} L^{\prime} \cos \gamma$ where $\gamma$ is the angle between the vertical and the normal to the upper beam surface i.e. $\gamma=$ $\tan ^{-1}\left(\frac{\left(h_{1}-h_{2}\right)}{2 L}\right)$ and $L^{\prime}$, the length of the upper surface is $L^{\prime}=L / \cos \gamma$. As such, the intensity of the line load on the beam is $P_{0}$.

\footnotetext{
‡ The dimension of the beam perpendicular to the plane on which it is drawn is assumed unity.
} 
The temporal pulse load is defined as:

$$
P(t)=\left\{\begin{array}{lr}
P_{0} & 0<t \leq t d \\
0 & t>t d
\end{array}\right.
$$

Finite element models have been set up and run using ABAQUS 6.14-2. The model has been meshed using a fine mesh of 0.25 element size and quadratic standard/implicit plane stress elements of type CPS8R, which is an 8-noded biquadratic plane stress quadrilateral, reduced integration element. The ratio of artificial to strain energy is almost zero which ensures no hour glassing spurious zero energy modes are present in the model. Displacement time histories have been recorded at 8 equidistant points along the length of the beam's neutral axis, with point 9 being at the boundary and point 1 at the tip, and the results are correlated with those of the analytical model proposed.

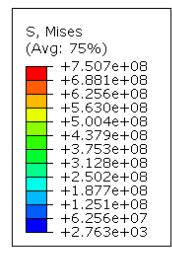

Figure 6: Contour of von Mises stress in the vibrating non-prismatic beam

For the sake of the study in this section $P_{0}=10^{6} \mathrm{~N}$ and $t_{d}=1.6 \mathrm{sec}$.

An implicit numerical integration scheme has been used to derive the numerical solution to the linear system of coupled ODE's, however; other methods such as Laplace Transform method or mode superposition are also applicable. Fig. 7 shows the comparison of displacements at two different (sample) points on the beam axis obtained by ABAQUS and the analytical model. Fig. 8 shows displacements at 8 points obtained by ABAQUS and Fig. 9 presents the same data obtained by the analytical model. It can be shown that by including 5 terms in the approximation of the displacement and rotation fields in the analytical model the results converge to those obtained by finite element analyses. This is especially true of the maximum displacements as in a pulse loaded scenario one is mostly interested in maxima of response parameters as displacements, strains, or stresses rather than detailed time history of these parameters. Maximum displacements have been corroborated and the results are depicted in Fig. 10 and as it can be seen the correlation is strong. It must be mentioned that Fig. 10 does not show the profile of deformation but rather the envelope of the response. This means maximum displacements at different locations along the beam do not necessarily happen at exactly the same time. 

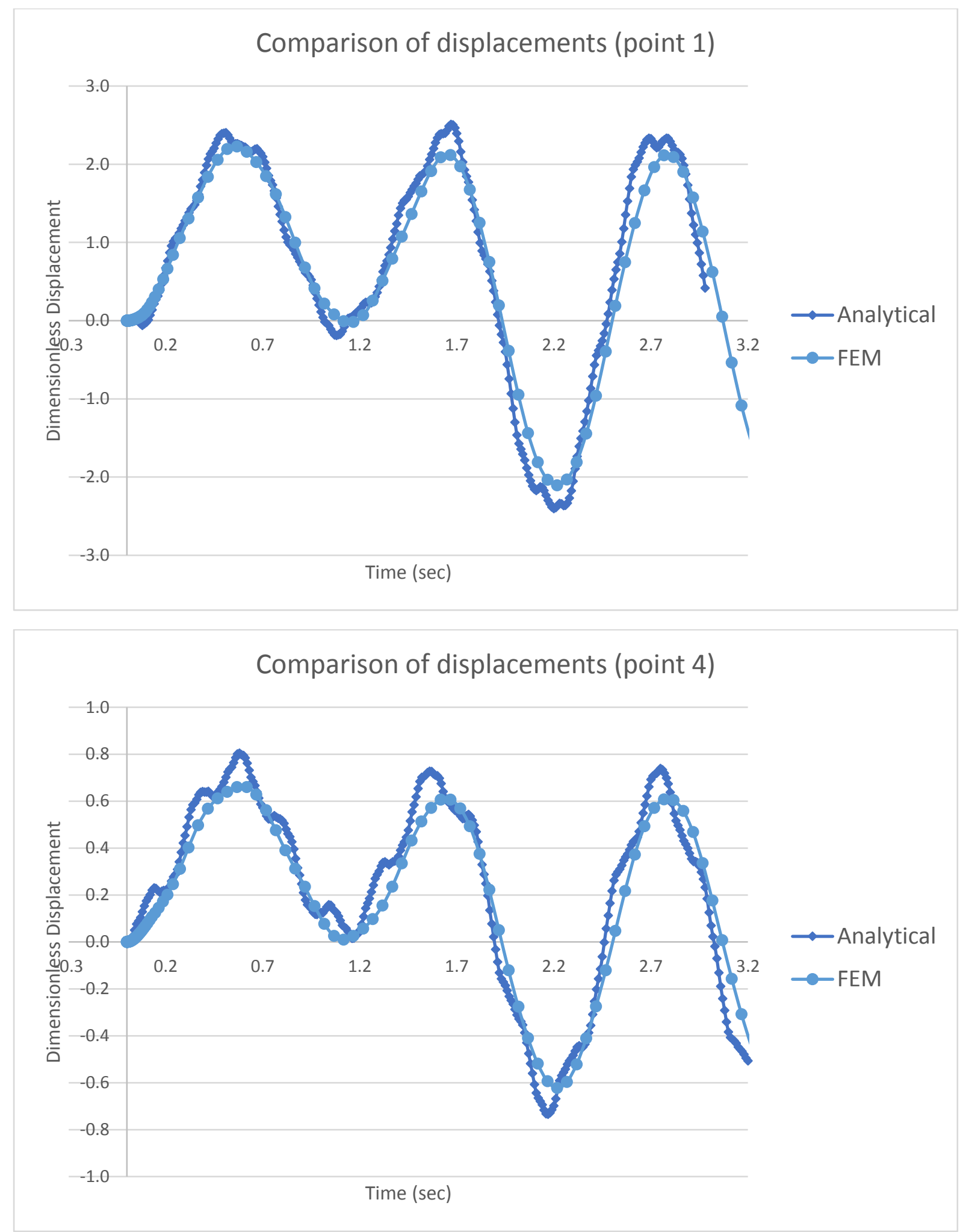

Figure 7: Comparison of displacement time histories for the beam at two sample points 


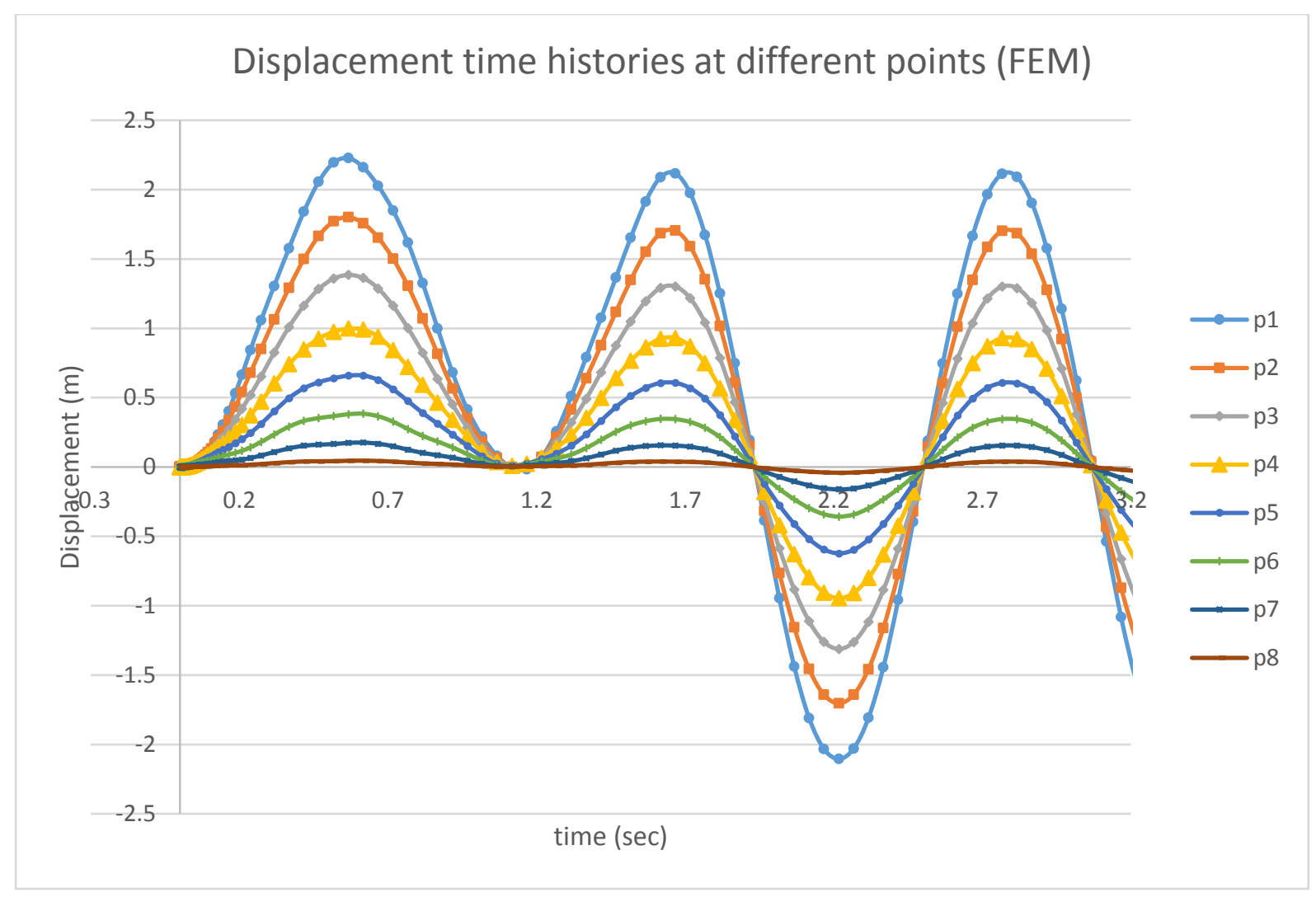

Figure 8: Displacement time histories at different points obtained using FEM

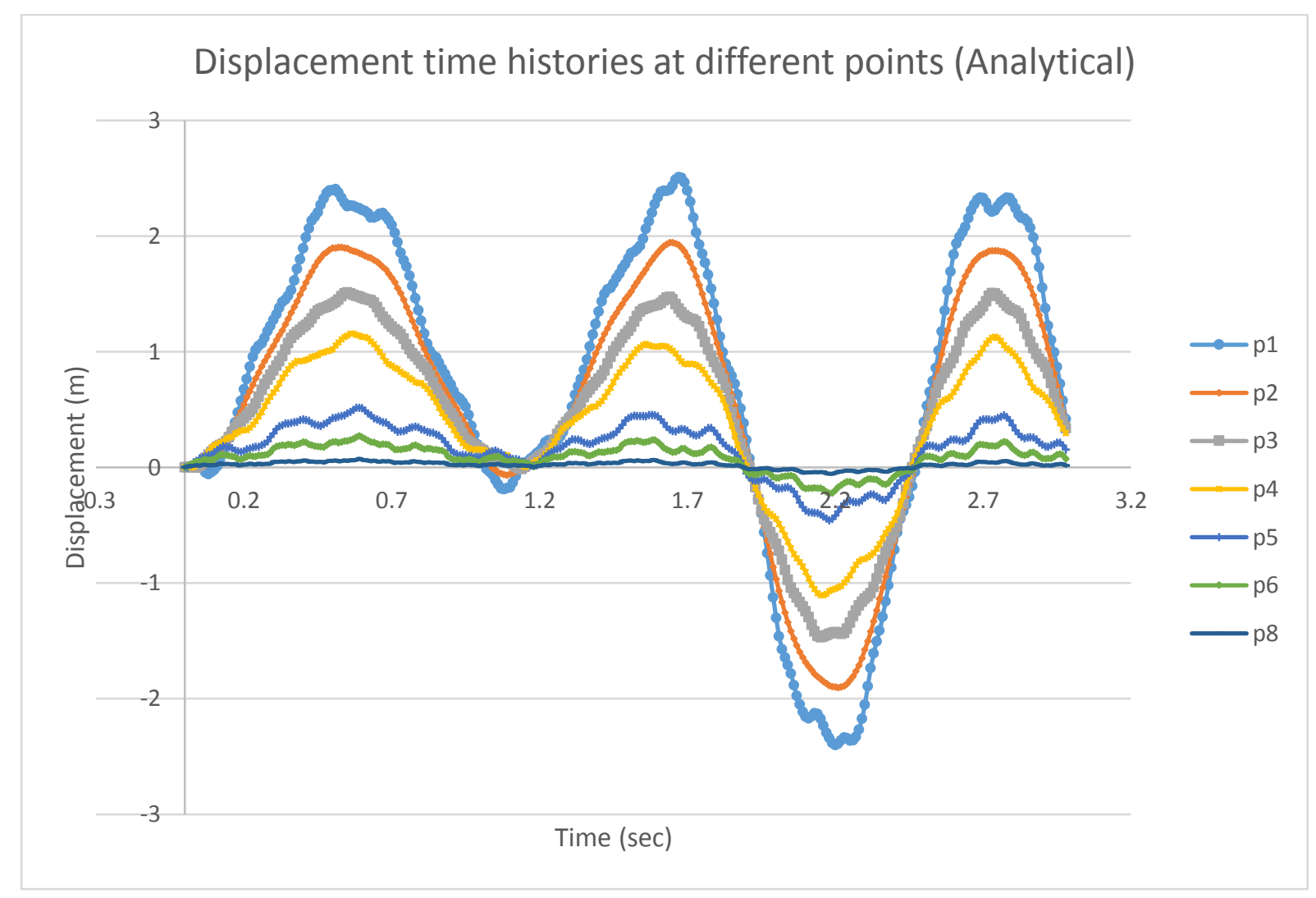

Figure 9: Displacement time histories at different points obtained using the analytical model 


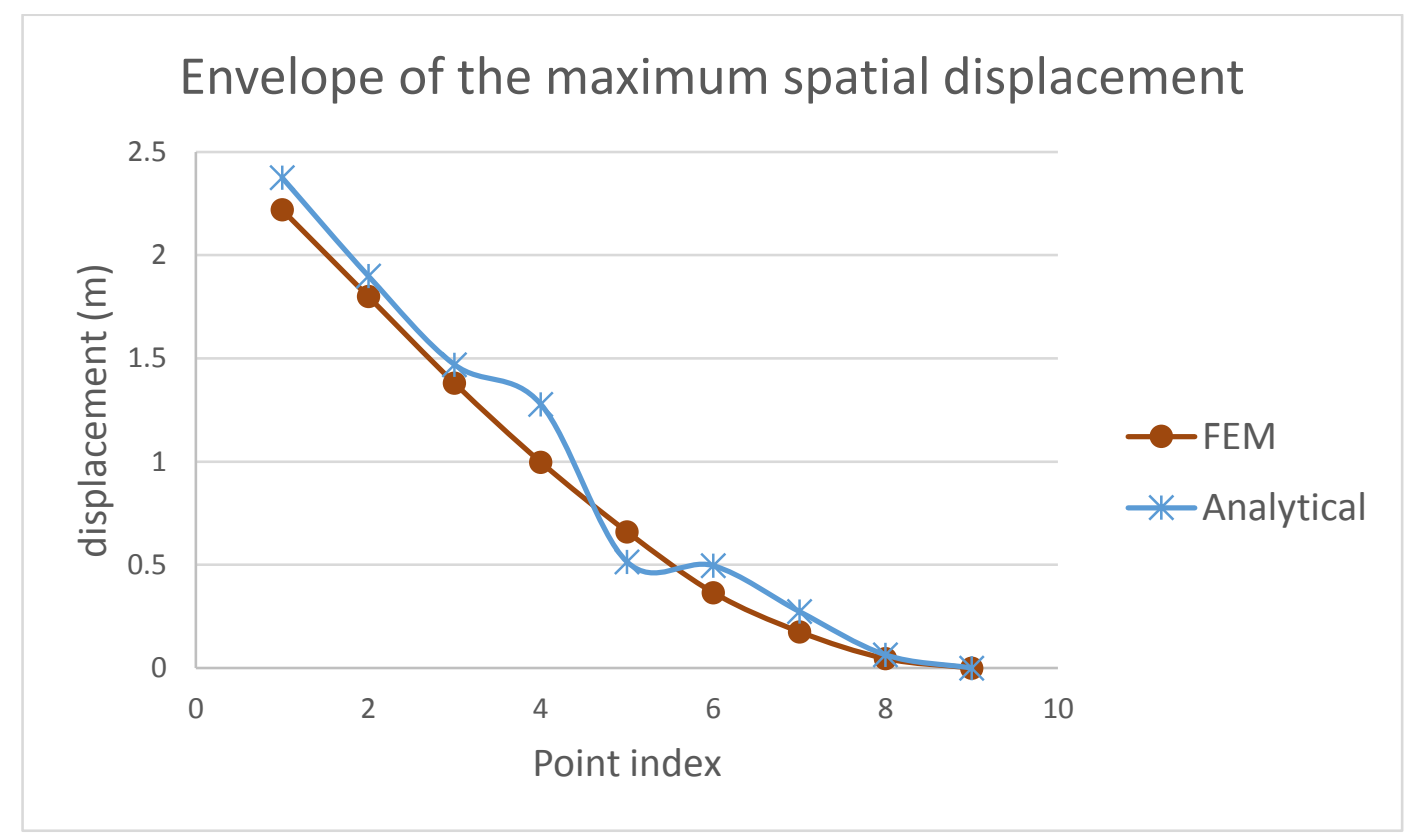

Figure 10: Comparison of maximum displacements

It is possible to obtain non-dimensional response parameters. This particularly proves useful if one were to conduct parametric studies on the influence of different input parameters on the output. The effect of different parameters on frequency were studied by researchers (see [32-34], for example).

\subsection{Non-dimensional response parameters}

Parametric studies are not conducted in the present work, however; the dimensionless parameters for the response are constructed using Buckingham's Pi-theorem. The reason is, up to this point, most equations have been presented using dimensionless parameters so it would be good practice if we pursued along this line. Dimensional analyses could be conducted in different ways. In one approach, all equations can be non-dimensionalised [35]. This requires the time as well as the spatial coordinate be non-dimensional. This method is of special interest in wave propagation and transient problems. The dimensionless spatial coordinate has already been introduced as $\xi=x / L$. Time can likewise be nondimensionalised using the wave speed in the medium and a single characteristic length e.g. the length of the beam as $\tau=t / L \sqrt{\frac{E}{\rho}}$. Any other dimension (e.g. $h_{1}$ or $h_{2}$ ) could have been used to derive the dimensionless time $\tau$. The important point is all equations could be nondimensionalised by keeping in mind $\frac{d \aleph}{d \tau}=\dot{\aleph} L \sqrt{\frac{\rho}{E}}$ and by extension $\frac{d^{2} \aleph}{d \tau^{2}}=\ddot{\aleph} L^{2} \rho / E$ where $\dot{O}=$ $d() / d t$.

An alternative approach involves keeping the governing differential equations dimensional and merely presenting the response and input parameters as non-dimensional. This will generalise the solutions and well as providing conclusions regarding parameters on which parametric studies have not been directly applied. This renders the conclusions more general 
and avoids repetition in generating points already generated in the dimensionless space. As input parameters have already been presented in dimensionless form the dimensionless response parameters are derived as follows:

$$
\begin{aligned}
& \frac{W_{0}}{L}=f\left(\frac{h_{1}}{L}, \frac{h_{1}}{h_{2}}, \frac{t_{d}}{L} \sqrt{\frac{E}{\rho}}, \frac{P_{0}}{E}\right), \\
& \frac{t_{m}}{t_{d}}=g\left(\frac{h_{1}}{L}, \frac{h_{1}}{h_{2}}, \frac{t_{d}}{L} \sqrt{\frac{E}{\rho}}, \frac{P_{0}}{E}\right) .
\end{aligned}
$$

Where in Eq.'s (34a) and (34b) $W_{0}$ is the maximum dynamic displacement of the beam (or the displacement at a particular point along the beam) and $t_{m}$ is the time at which that maximum is attained. $f, g, p$ and $q$ denote generic functions and for a homogeneous non-prismatic beam they are smooth in general. Alternative formulations are also possible as follows:

$$
\begin{gathered}
\frac{\sigma_{m}}{P_{0}}=p\left(\frac{I_{1}}{h_{2}^{3}}, \frac{h_{1}}{L}, \frac{t_{d}}{L} \sqrt{\frac{E}{\rho}}, \frac{P_{0}}{E}\right), \\
\varepsilon_{m}=p\left(\frac{I_{1}}{h_{2}^{3}}, \frac{h_{1}}{L}, \frac{t_{d}}{L} \sqrt{\frac{E}{\rho}}, \frac{P_{0}}{E}\right) .
\end{gathered}
$$

Where $\sigma_{m}$ signifies the maximum stress $\left(\sigma_{m}=\max _{t, x}\left(\sigma_{i j}\right)\right)$ and the maximum strain $\varepsilon_{m}$ in the beam and $I_{1}=\frac{h_{1}^{3}}{12}$. For an arbitrary pulse shape the pulse can be represented by its normalised temporal function of shape (see Fig. 11), its maximum $P_{0}$ and duration $t_{d}$. For the case of an impulsive load the shape of the pressure time-history is irrelevant and the total impulse $I_{m}=\int_{0}^{t_{d}} P(t) d t$ determines the response. In such a scenario the independent input parameters $\frac{t_{d}}{L} \sqrt{\frac{E}{\rho}}$ and $\frac{P_{0}}{E}$ are replaced by a single parameter $\frac{I_{m}}{L \sqrt{E \rho}}$. This is shown in Eq. (36) for a generic dimensionless response parameter $\pi_{i}$.

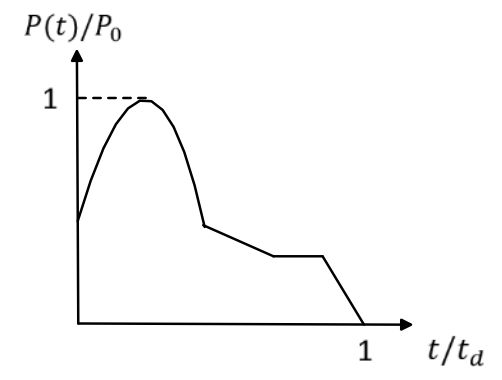

Fig. 11: Normalised arbitrary temporal pulse shape

$$
\pi_{i}=\pi_{i}\left(\frac{h_{1}}{L}, \frac{h_{1}}{h_{2}}, \frac{I_{m}}{L \sqrt{E \rho}}\right)
$$




\section{Conclusions}

The current study deals with evaluation of the dynamic response of homogeneous nonprismatic Timoshenko cantilever beams under lateral pulse loading. Following the derivation of the equations of motion using the extended principle of least action (extended Hamilton's principle) the eigenvalue problem has been set up by equating the excitation function to zero. The coupled system of non-dimensional equations of motion are decoupled and expressed in terms of displacement or rotation, only, following the approach presented in [28].

The eigenvalues and eigenfunctions are then obtained through analyses of the system. A complete set of functions obtained by researchers has been used to derive the eigenfunctions of the non-prismatic beam. As the set consists of functions satisfying the essential boundary conditions and is a complete set, any displacement function can be expressed as a linear combination of them. The dynamic response under pulse loading is obtained using the method of eigenfunctions expansion which attributes to displacement and rotation fields generalised coordinates when shape functions are the exact modes.

The results of dynamic analyses are presented for a given spatial and temporal distribution of the dynamic pulse and are corroborated with those obtained by commercial FE software ABAQUS 6.14-2. Error in the estimation of eigenfrequencies is bounded to $10 \%$ for the first 8 modes. Maximum dynamic displacement also correlates well with FE results.

Although the study encompasses analysis of the Timoshenko beam as it includes both the effects of rotatory inertia and shear deformation, it is possible, through the deletion of terms associated with each effect, to obtain the other three models explained in Appendix A.

Dimensionless parameters of response are introduced and the two methods of nondimensionalisation are explained which could be useful in dealing with generic problems of a particular formulation. While the first approach proposes non-dimensional partial differential equations to be solved the second approach makes use of the fact that the non-dimensional response is a function of non-dimensional input parameters, only. Both approaches are valid and could be pursued.

It is worth noting a parallel formulation of the same problem contains frequency domain analyses where the input and output are mapped through Fourier complex transformation and the modal complex frequency response functions are used to determine the response. This line was not pursued in the present study but has some advantages as multiplicative nature of response terms which renders the solution, in principle, more easily obtainable.

A final remark here on the accuracy of models in prediction of out-of-plane shear stress is in order. All the theories considered give incorrect results for out-of-plane shear at the top and bottom of the beam cross section. Third-order shear deformation theory, on the other hand, deals with this problem adequately and provides a solution satisfying the zero shear stress on the free surfaces. It is, in particular, useful in the study of thick laminated composite plates and it could be shown that if functionally graded materials are not of concern it is the exact solution. 


\section{Acknowledgements}

The authors wish to express their gratitude for the financial support provided by TurkAz Enerji Ltd. under contract No. AESZ P56658.

Bibliography:

1. Auclair, S.C., et al., The effect of rotatory inertia on the natural frequencies of composite beams. Journal of Sound and Vibration, 2016. 366: p. 230-247.

2. Han, S.M., H. Benaroya, and T. Wei, Dynamics of transversely vibrating beams using four engineering theories. Journal of Sound and Vibration, 1999. 225(5): p. 935-988.

3. Lee, D.G., et al., Novel micro vibration energy harvesting device using frequency up conversion. Transducers '07 \& Eurosensors Xxi, Digest of Technical Papers, Vols 1 and 2, 2007: p. U440U441.

4. Berman, G.P., et al., Spin relaxation caused by thermal excitations of high-frequency modes of cantilever vibrations. Physical Review B, 2003. 68(9).

5. Berman, G.P., V.N. Gorshkov, and V.I. Tsifrinovich, Reduction of magnetic noise in magnetic resonance force microscopy. Physical Review B, 2004. 69(21).

6. Ruta, P., The vibration of a non-prismatic beam on an inertial elastic half-plane. Journal of Sound and Vibration, 2004. 275(3-5): p. 533-556.

7. Wang, G.F. and X.Q. Feng, Timoshenko beam model for buckling and vibration of nanowires with surface effects. Journal of Physics D-Applied Physics, 2009. 42(15).

8. Wang, C.M., V.B.C. Tan, and Y.Y. Zhang, Timoshenko beam model for vibration analysis of multi-walled carbon nanotubes. Journal of Sound and Vibration, 2006. 294(4-5): p. 1060-1072.

9. Lin, S.C. and K.M. Hsiao, Vibration analysis of a rotating Timoshenko beam. Journal of Sound and Vibration, 2001. 240(2): p. 303-322.

10. Rao, S.S. and R.S. Gupta, Finite element vibration analysis of rotating Timoshenko beams. Journal of Sound and Vibration, 2001. 242(1): p. 103-124.

11. Yardimoglu, B., Vibration analysis of rotating tapered Timoshenko beams by a new finite element model. Shock and Vibration, 2006. 13(2): p. 117-126.

12. Bazoune, A. and Y.A. Khulief, A Finite Beam Element for Vibration Analysis of Rotating Tapered Timoshenko Beams. Journal of Sound and Vibration, 1992. 156(1): p. 141-164.

13. Karabalis, D.L. and D.E. Beskos, Static, Dynamic and Stability Analysis of Structures Composed of Tapered Beams. Computers \& Structures, 1983. 16(6): p. 731-748.

14. Keller, T., T. Tirelli, and A.X. Zhou, Tensile fatigue performance of pultruded glass fiber reinforced polymer profiles. Composite Structures, 2005. 68(2): p. 235-245.

15. Kuo, Y.H., T.H. Wu, and S.Y. Lee, Bending Vibrations of a Rotating Nonuniform Beam with Tip Mass and an Elastically Restrained Root. Computers \& Structures, 1992. 42(2): p. 229-236.

16. Rossi, R.E. and P.A.A. Laura, Numerical Experiments on Vibrating, Linearly Tapered Timoshenko Beams. Journal of Sound and Vibration, 1993. 168(1): p. 179-183.

17. Yardimoglu, B., A novel finite element model for vibration analysis of rotating tapered Timoshenko beam of equal strength. Finite Elements in Analysis and Design, 2010. 46(10): p. 838-842.

18. Attarnejad, R., S.J. Semnani, and A. Shahba, Basic displacement functions for free vibration analysis of non-prismatic Timoshenko beams. Finite Elements in Analysis and Design, 2010. 46(10): p. 916-929.

19. Attarnejad, R., A. Shahba, and M. Eslaminia, Dynamic basic displacement functions for free vibration analysis of tapered beams. Journal of Vibration and Control, 2011. 17(14): p. 22222238. 
20. Attarnejad, R., A. Shahba, and S.J. Semnani, Analysis of Non-Prismatic Timoshenko Beams Using Basic Displacement Functions. Advances in Structural Engineering, 2011. 14(2): p. 319332.

21. Finlayson, B.A., Citation Classic - the Method of Weighted Residuals - a Review. Current Contents/Engineering Technology \& Applied Sciences, 1983(40): p. 22-22.

22. Laura, P.A.A., et al., Numerical Experiments on Free and Forced Vibrations of Beams of NonUniform Cross-Section. Journal of Sound and Vibration, 1988. 120(3): p. 587-596.

23. Pachenari, Z. and R. Attarnejad, Analysis of Tapered Thin Plates Using Basic Displacement Functions. Arabian Journal for Science and Engineering, 2014. 39(12): p. 8691-8708.

24. Kane, J.H., B.L.K. Kumar, and R.H. Gallagher, Boundary-Element Direct Reanalysis for Continuum Structures. Journal of Engineering Mechanics-Asce, 1992. 118(8): p. 1679-1691.

25. Chu, F.H. and W.D. Pilkey, Transient Analysis of Structural Members by the Csdt Riccati Transfer-Matrix Method. Computers \& Structures, 1979. 10(4): p. 599-611.

26. Lee, S.Y., H.Y. Ke, and Y.H. Kuo, Analysis of Nonuniform Beam Vibration. Journal of Sound and Vibration, 1990. 142(1): p. 15-29.

27. Just, D.J., Plane Frameworks of Tapering Box and I-Section. Journal of the Structural DivisionAsce, 1977. 103(1): p. 71-86.

28. Lee, S.Y. and S.M. Lin, Vibrations of Elastically Restrained Nonuniform Timoshenko Beams. Journal of Sound and Vibration, 1995. 184(3): p. 403-415.

29. Landau, L.D., et al., Theory of elasticity. 3rd English ed. Course of theoretical physics. 1986, Oxford Oxfordshire ; New York: Pergamon Press. viii, $187 \mathrm{p}$.

30. Buckingham, E., On Physically Similar Systems; Illustrations of the Use of Dimensional Equations. Physical Review, 1914. 4(4): p. 345-376.

31. Lee, S.Y. and Y.H. Kuo, Exact-Solutions for the Analysis of General Elastically Restrained Nonuniform Beams. Journal of Applied Mechanics-Transactions of the Asme, 1992. 59(2): p. S205-S212.

32. Lee, H.L. and W.J. Chang, Surface and small-scale effects on vibration analysis of a nonuniform nanocantilever beam. Physica E-Low-Dimensional Systems \& Nanostructures, 2010. 43(1): p. 466-469.

33. Lee, S.Y. and J.C. Chao, Exact solutions for out-of-plane vibration of curved nonuniform beams. Journal of Applied Mechanics-Transactions of the Asme, 2001. 68(2): p. 186-191.

34. Lee, S.Y. and J.Y. Hsiao, Free in-plane vibrations of curved nonuniform beams. Acta Mechanica, 2002. 155(3-4): p. 173-189.

35. Langhaar, H.L., Dimensional analysis and theory of models. 1980, Huntington, N.Y.: R. E. Krieger Pub. Co. xi, 166 p.

\section{Appendix A}

The most commonly used beam theories are as follows:

1. Euler-Bernoulli beam model:

In this case the kinetic energy $T$ in the system is due to flexural displacement filed $w(x, t)$ (or rather transverse velocity field $\dot{w}(x, t))$ and the strain energy $U_{b}$ is only due to bending (curvature field).

$$
U=U_{b}=\frac{1}{2} \int_{0}^{L} E I(x)\left(\frac{\partial^{2} w}{\partial x^{2}}\right)^{2} d x
$$




$$
T=T_{t}=\frac{1}{2} \int_{0}^{L} \rho A(x)\left(\frac{\partial w}{\partial t}\right)^{2} d x
$$

And the work of non-conservative forces as follows:

$$
\delta W_{n c}=\int_{0}^{L} f(x, t) \delta w d x
$$

Thus the principle of least action yields the following:

$$
\delta S=\int_{t_{1}}^{t_{2}} \delta(T-U) d t+\int_{t_{1}}^{t_{2}} \delta W_{n c} d t=0
$$

Which implies:

$$
\begin{gathered}
\delta S=\delta \int_{t_{1}}^{t_{2}}\left\{\int_{0}^{L} \frac{1}{2} \rho A(x)\left(\frac{\partial w}{\partial t}\right)^{2} d x-\int_{0}^{L} \frac{1}{2} E I(x)\left(\frac{\partial^{2} w}{\partial x^{2}}\right)^{2} d x\right\} d t+\int_{t_{1}}^{t_{2}} \int_{0}^{L} f(x, t) \delta w d x d t \\
=0 \\
\delta S=\int_{t_{1}}^{t_{2}} \int_{0}^{L}\left(\rho A(x) \dot{w} \delta \dot{w}-E I(x) w^{\prime \prime} \delta w^{\prime \prime}\right) d x d t+\int_{t_{1}}^{t_{2}} \int_{0}^{L} f(x, t) \delta w d x d t=0
\end{gathered}
$$

Given that on integration by parts the integrations involved yield the following

$$
\int_{0}^{L} E I(x) w^{\prime \prime} \delta w^{\prime \prime} d x=\left[\left(E I w^{\prime \prime}\right) \delta w^{\prime}\right]_{0}^{L}-\left[\left(E I w^{\prime \prime}\right)^{\prime} \delta w\right]_{0}^{L}+\int_{0}^{L}\left(E I(x) w^{\prime \prime}\right)^{\prime \prime} \delta w d x
$$

And

$$
\int_{t_{1}}^{t_{2}} \rho A(x) \dot{w} \delta \dot{w} d t=[\rho A(x) \dot{w} \delta w]_{t_{1}}^{t_{2}}-\int_{t_{1}}^{t_{2}} \rho A(x) \ddot{w} \delta w d t
$$

And knowing that the first two terms in Eq. (A.7) represent boundary conditions and the first term in Eq. (A.8) vanishes as a result of the fundamental assumption of Hamilton's principle the governing PDE (A.9) plus boundary conditions (A.10) and (A.11):

$$
\begin{aligned}
& \rho A(x) \ddot{w}+\left(E I(x) w^{\prime \prime}\right)^{\prime \prime}=f(x, t) \\
& {\left[\left(E I w^{\prime \prime}\right) \delta w^{\prime}\right]_{0}^{L}=0} \\
& {\left[\left(E I w^{\prime \prime}\right)^{\prime} \delta w\right]_{0}^{L}=0}
\end{aligned}
$$

Subsequent to the expansion of terms and rearrangement this yields:

$$
\rho A(x) \ddot{w}+E I(x) w^{(4)}+2(E I(x))^{\prime} w^{\prime \prime \prime}+(E I(x))^{\prime \prime} w^{\prime \prime}=f(x, t)
$$

So the eigenvalue problem is as follows:

$$
\rho A(x) \ddot{w}+E I(x) w^{(4)}+2(E I(x))^{\prime} w^{\prime \prime \prime}+(E I(x))^{\prime \prime} w^{\prime \prime}=0
$$

Boundary conditions for the cantilever beam are as follows: 


$$
\begin{aligned}
& w(0)=0, w^{\prime}(0)=0 \\
& E I w^{\prime \prime}(L)=0,\left(E I w^{\prime \prime}\right)^{\prime}(L)=0
\end{aligned}
$$

\section{Rayleigh beam model}

In this model the strain energy is, as in the Euler-Bernoulli beam model, due to bending only, but the kinetic energy depends upon both translational and rotational velocity fields.

$$
\begin{gathered}
U=U_{b}=\frac{1}{2} \int_{0}^{L} E I(x)\left(\frac{\partial^{2} w}{\partial x^{2}}\right)^{2} d x \\
T=T_{t}+T_{r}=\frac{1}{2} \int_{0}^{L} \rho A(x)\left(\frac{\partial w}{\partial t}\right)^{2} d x+\frac{1}{2} \int_{0}^{L} \rho I(x)\left(\frac{\partial^{2} w}{\partial x \partial t}\right)^{2} d x \\
\delta S=\int_{t_{1}}^{t_{2}} \int_{0}^{L}\left(\rho A(x) \dot{w} \delta \dot{w}+\rho I(x) \dot{w}^{\prime} \delta \dot{w}^{\prime}-E I(x) w^{\prime \prime} \delta w^{\prime \prime}\right) d x d t+\int_{t_{1}}^{t_{2}} \int_{0}^{L} f(x, t) \delta w d x d t \\
=0
\end{gathered}
$$

Given that:

$$
\begin{gathered}
\int_{t_{1}}^{t_{2}} \int_{0}^{L} \rho I(x) \dot{w}^{\prime} \delta \dot{w}^{\prime} d x d t=\int_{t_{1}}^{t_{2}}\left[\rho I(x) \dot{w}^{\prime} \delta \dot{w}^{\prime}\right]_{0}^{L}-\int_{t_{1}}^{t_{2}} \int_{0}^{L}\left(\rho I(x) \dot{w}^{\prime}\right)^{\prime} \delta \dot{w} d x d t \quad(A .19) \\
\delta S=\int_{t_{1}}^{t_{2}} \int_{0}^{L}\left(-\rho A(x) \ddot{w} \delta w-\left(\rho I(x) \dot{w}^{\prime}\right)^{\prime} \delta \dot{w}-\left(E I(x) w^{\prime \prime}\right)^{\prime \prime} \delta w\right) d x d t+\int_{t_{1}}^{t_{2}}\left[\rho I(x) \dot{w}^{\prime} \delta \dot{w}\right]_{0}^{L} \\
+\int_{0}^{L}[\rho A(x) \dot{w} \delta w]_{0}^{L} d x-\int_{t_{1}}^{t_{2}}\left[\left(E I(x) w^{\prime \prime}\right) \delta w^{\prime}\right]_{0}^{L} d t \\
+\int_{t_{1}}^{t_{2}}\left[\left(E I(x) w^{\prime \prime}\right)^{\prime} \delta w\right]_{0}^{L} d t+\int_{t_{1}}^{t_{2}} \int_{0}^{L} f(x, t) \delta w d x d t \\
=0
\end{gathered}
$$

Which considering the zero terms after integration by parts yields:

$$
\begin{gathered}
\rho A(x) \ddot{w}-\left(\rho I(x) \ddot{w}^{\prime}\right)^{\prime}+\left(E I(x) w^{\prime \prime}\right)^{\prime \prime}=f(x, t) \\
{\left[\left(\left(E I w^{\prime \prime}\right)^{\prime}-\rho I \ddot{w}^{\prime}\right) \delta w\right]_{0}^{L}=0 \quad(A .22)} \\
{\left[\left(E I w^{\prime \prime}\right) \delta w^{\prime}\right]_{0}^{L}=0 \quad(A .23)}
\end{gathered}
$$

\section{Shear beam model}

In this case the strain energy in the beam is due to both bending flexure and shear distortion and is expressed as $U=U_{b}+U_{s}$.

Defining the new kinematic variables of $\alpha(x, t)$ as the angle of rotation of the section and $\beta(x, t)$ as the angle of distortion due to shear, energy terms are redefined as following: 


$$
\begin{gathered}
U_{b}=\frac{1}{2} \int_{0}^{L} E I(x)\left(\frac{\partial \alpha}{\partial x}\right)^{2} d x=\frac{1}{2} \int_{0}^{L} E I(x) \alpha^{\prime 2} d x \\
U_{s}=\frac{1}{2} \int_{0}^{L} k G A(x) \beta^{2} d x=\frac{1}{2} \int_{0}^{L} k G A(x)\left(\frac{\partial w}{\partial x}-\alpha\right)^{2} d x \\
T=T_{t}=\frac{1}{2} \int_{0}^{L} \rho A(x)\left(\frac{\partial w}{\partial t}\right)^{2} d x \quad(\text { A. 26) }
\end{gathered}
$$

Notice the relation below is approximately valid for the beam:

$$
\alpha(x, t)+\beta(x, t)=\frac{\partial w}{\partial x}
$$

Thus the principle of least action yields the following

$$
\begin{gathered}
\delta S=\int_{t_{1}}^{t_{2}} \int_{0}^{L}\left(\rho A(x) \dot{w} \delta \dot{w}-k G A(x)\left(w^{\prime}-\alpha\right)\left(\delta w^{\prime}-\delta \alpha\right)-E I(x) w^{\prime \prime} \delta w^{\prime \prime}\right) d x d t \\
+\int_{t_{1}}^{t_{2}} \int_{0}^{L} f(x, t) \delta w d x d t=0 \quad(A .28)
\end{gathered}
$$

On integration by parts and omitting the terms which yield zero one can obtain the following governing PDE's along with boundary conditions:

$$
\begin{aligned}
& \rho A(x) \ddot{w}+\left[k G A(x)\left(\alpha-w^{\prime}\right)\right]^{\prime}=f(x, t) \\
& k G A(x)\left(\alpha-w^{\prime}\right)-\left(E I \alpha^{\prime}\right)^{\prime}=0 \\
& {\left[k G A(x)\left(\alpha-w^{\prime}\right) \delta w\right]_{0}^{L}=0} \\
& {\left[E I \alpha^{\prime} \delta \alpha\right]_{0}^{L}=0}
\end{aligned}
$$

\section{Appendix B}

The dimensionless frequency is defined by Eq. (10g) as follows:

$$
\Omega^{2}=\rho A(0) \omega^{2} L^{4} / E I(0)(10 g)
$$

Considering parameters $\delta$ and $\eta$ defined by Eq.'s (10e) and (10f), respectively; one can derive the following:

$$
\begin{aligned}
\delta & =E I(0) /\left(k G A(0) L^{2}\right) \\
\eta & =J(0) /\left[\rho A(0) L^{2}\right] \\
\delta \eta & =E I^{2}(0) /\left(k G A^{2}(0) L^{4}\right)
\end{aligned}
$$


Which renders $\Omega_{c}^{2}=\frac{1}{\delta \eta} \propto \frac{L^{4}}{\frac{I^{2}}{A^{2}}}=\lambda^{4}$, where is the slenderness defined as $\lambda=\frac{L}{\sqrt{\frac{I}{A}}}$, and $\sqrt{\frac{I}{A}}$ is the radius of gyration. It is obvious that:

$$
\lim _{\lambda \rightarrow 0} \Omega_{c}^{2}=0 \text { and } \lim _{\lambda \rightarrow+\infty} \Omega_{c}^{2}=+\infty
$$

\section{Appendix C}

Let $V_{i}$ represent a complete set of linearly independent orthonormal functions. By the virtue of orthonormality Eq. (C.1) is satisfied (all functions are understood to be functions of a single spatial coordinate).

$$
\int_{\Omega} V_{i} V_{j} d \Omega=\delta_{i j}
$$

Where $\delta_{i j}$ is the Kronecker delta function. Any other function can be described as a linear combination of thus could be written as follows:

$$
\psi_{i}=a_{k}^{(i)} V_{k}
$$

Where Einstein's summation convention in implied on subscripts. Thus:

$$
\int_{\Omega} \psi_{i} \psi_{j} d \Omega=\int_{\Omega} a_{k}^{(i)} V_{k} a_{m}^{(j)} V_{m} d \Omega=a_{k}^{(i)} a_{m}^{(j)} \int_{\Omega} V_{k} V_{m} d \Omega=a_{k}^{(i)} a_{m}^{(j)} \delta_{k m}=a_{k}^{(i)} a_{k}^{(j)}
$$

Since there is no precondition on the Orthogonality of $\boldsymbol{a}^{(i)}$ and $\boldsymbol{a}^{(j)}$ the dot product of these vectors in general does not vanish.

$$
\boldsymbol{a}^{(i)} \cdot \boldsymbol{a}^{(j)}=a_{k}^{(i)} a_{k}^{(j)} \neq 0
$$

